\title{
Ag Nanocrystal Junctions as the Site for Surface-Enhanced Raman Scattering of Single Rhodamine 6G Molecules
}

\author{
Amy M. Michaels, Jiang Jiang, and Louis Brus* \\ Department of Chemistry, Columbia University, New York, New York 10027
}

Received: July 18, 2000; In Final Form: October 3, 2000

\begin{abstract}
Atomic force microscopy (AFM) measurements show that the Ag nanoparticles that yield surface-enhanced Raman scattering (SERS) of single molecules of Rhodamine (R6G) are all compact aggregates consisting of a minimum of two individual particles. Comparison of 514.5 and $632.8 \mathrm{~nm}$ excitation shows that the single molecule R6G signal is significantly higher when the excitation wavelength is resonant with the absorption band of R6G and suggests that the Raman excitation spectrum follows the absorption profile for R6G. We have also observed an interesting superlinear power dependence of the SERS signal. On average, by increasing the incident power by 2 orders of magnitude and decreasing the integration time by the same factor to maintain constant fluence, increases of 4 to 6 times were observed in the SERS intensity. We discuss these results in terms of model where the R6G molecule that yields single molecule SERS signals is located at the junction of two Ag nanoparticles. We have also modeled the system using molecular resonance Raman theory to provide further insight into the enhancement mechanism.
\end{abstract}

\section{Introduction}

Surface-enhanced Raman scattering (SERS) is a wellestablished phenomenon which can enhance Raman signals of nonresonant molecules adsorbed on noble metal particles by 5 to 6 orders of magnitude. ${ }^{1-4}$ In certain cases, however, the enhancement can be enormous. Several experiments have demonstrated that the SERS enhancement can approach $10^{14}$ to $10^{15}$, and, in fact, enable the detection of a single molecule..$^{5-10}$ Remarkably, the strength of the single molecule SERS signal can be much stronger than the luminescence signal from a single highly fluorescent molecule in the absence of metal.

We recently reported that the average single molecule SERS cross section for the dye Rhodamine $6 \mathrm{G}$ (R6G) is $\sim 200 \AA^{2}$ for certain "hot" SERS-active nanocrystals. This cross section includes both sharp Raman lines and broad underlying continuum. These SERS-active nanocrystals account for less than $1 \%$ of the total particles in a heterogeneous Ag colloid. Since the intensity of resonant Rayleigh scattering from a metal particle is also a measure of the average local field enhancement around the nanocrystal, we independently characterized the SERS and resonant Rayleigh scattering spectra from individual nanocrystals in order to probe the electromagnetic (EM) contribution to the SERS signal. ${ }^{11}$ These experiments demonstrated no direct correlation between the strength or frequency of the Rayleigh scattering resonance and the single molecule SERS activity of the individual Ag particles. Two characteristics common to all scattering spectra of SERS-active particles included (1) partial resonance at the SERS excitation wavelength, and (2) a complex scattering spectrum, consisting of at least 2 resonances. These results suggest that some other factor, not simply an intense plasmon resonance, is critical in distinguishing SERS-active particles. We interpreted these data in a framework originally proposed by both Otto and Persson, which considers the interaction of ballistic electrons in the metal with a strongly chemisorbed molecule. ${ }^{12,13}$ The number of SERS-

* Author to whom correspondence should be addressed. active particles is not limited by the number of available R6G molecules; increasing the R6G concentration by a factor of one hundred increases the number of SERS-active particles only by a factor of 5. This suggests that a rare chemisorption site may be the critical and limiting factor for single molecule SERS.

The possibility of a rare site was first discussed in prior ensemble colloidal SERS studies, where the giant SERS signal saturated at nanomolar concentrations of R6G that correspond to roughly one adsorbed molecule per $55 \mathrm{~nm}$ Ag particle. ${ }^{5}$ This rare site was characterized to have a very high adsorption energy of $65.9 \mathrm{~kJ} / \mathrm{mol}(\sim 2 / 3 \mathrm{eV})$. This was in contrast to a second site which had a lower adsorption energy of $35.8 \mathrm{~kJ} / \mathrm{mol}$ and yielded SERS enhancements that were weaker by more than 2 orders of magnitude.

This article presents AFM measurements which demonstrate that the SERS-active particles are compact aggregates consisting of two or more $50 \mathrm{~nm} \mathrm{Ag}$ particles. On the basis of these results, we propose that the rare site required for single molecule SERS lies at the junction of two aggregated particles. We also observe an unusual power dependence in that the scattering is higher than linear for most, but not all, SERS-active aggregates. We have also modeled the nanocrystal/molecule complex within the framework of resonance Raman theory in order to gain further insight into the enhancement mechanism.

\section{Experimental Section}

Sample preparation and the microscope-based optical imaging apparatus have been described in detail previously. ${ }^{11}$ Briefly, the sample was prepared by incubating a nanomolar concentration of R6G in a colloidal silver solution containing $1-10 \mathrm{mM}$ $\mathrm{NaCl}$ for $\sim 2 \mathrm{~h}$. The resulting solution was diluted by a factor of 3 with a boiled sodium citrate solution, and then spin-cast onto a polylysine-coated quartz cover slip. For AFM studies, the solution was similarly diluted by a factor of 30 . Studies were conducted at room temperature in air. Resonant Rayleigh spectra were obtained using the microscope equipped with a 

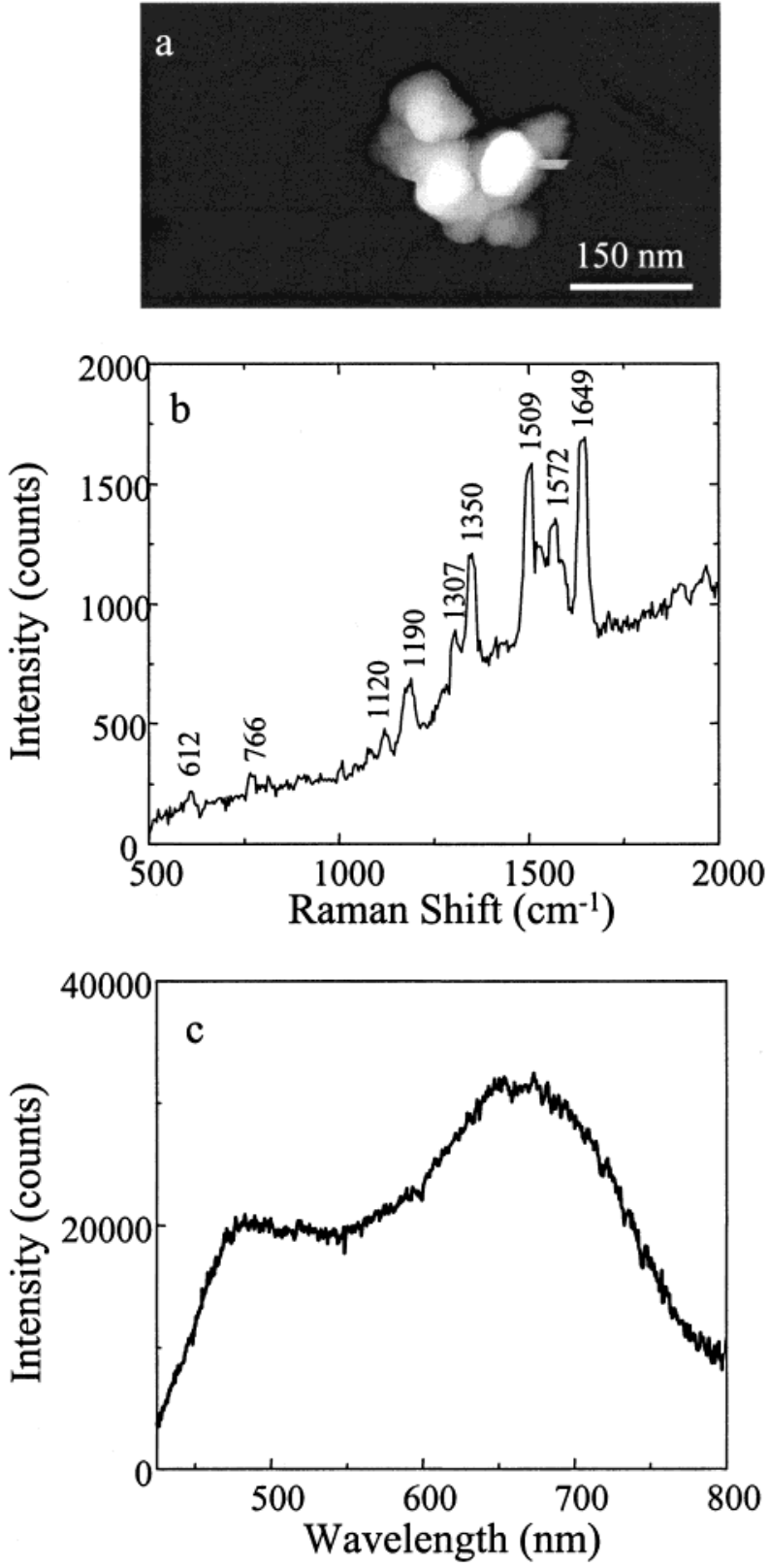

Figure 1. (a) AFM image, (b) R6G SERS spectrum, and (c) resonant Rayleigh scattering spectrum of a single SERS-active Ag particle incubated with $10 \mathrm{mM} \mathrm{NaCl}$ and $2 \mathrm{nM} \mathrm{R6G}$. For the SERS spectrum, the intergration time was $60 \mathrm{~s}$ at $\sim 30 \mathrm{~W} / \mathrm{cm}^{2}$. The Rayleigh scattering spectrum was obtained with a $10 \mathrm{~s}$ integration time.

dark-field condenser and a tungsten lamp. For SERS measurements, the sample was irradiated with $\sim 35 \mathrm{~mW}$ of either continuous wave $514.5 \mathrm{~nm}$ (Coherent Innova 308) or $632.8 \mathrm{~nm}$ (Coherent High Power HeNe) laser light in grazing incidence geometry, yielding a power density of $\sim 30 \mathrm{~W} / \mathrm{cm}^{2}$. In the power dependence experiments, samples were illuminated by the laser at $3 \mathrm{~W} / \mathrm{cm}^{2}, 30 \mathrm{~W} / \mathrm{cm}^{2}$, and $300 \mathrm{~W} / \mathrm{cm}^{2}$, while the integration time was adjusted from $10 \mathrm{~s}$ to $1 \mathrm{~s}$ to $0.1 \mathrm{~s}$, respectively. The linearity of the instrumental response under the same conditions (i.e., integration time and power) was calibrated by observation of laser scattering from imperfections in bare coverslips. To obtain AFM images, the SERS-active particles were first identified optically, and the dark-field optics were then removed from the microscope. The AFM scanner (Digital Instruments, Bioscope) was mounted on the sample stage and the SERSactive particles were identified and imaged in tapping mode.
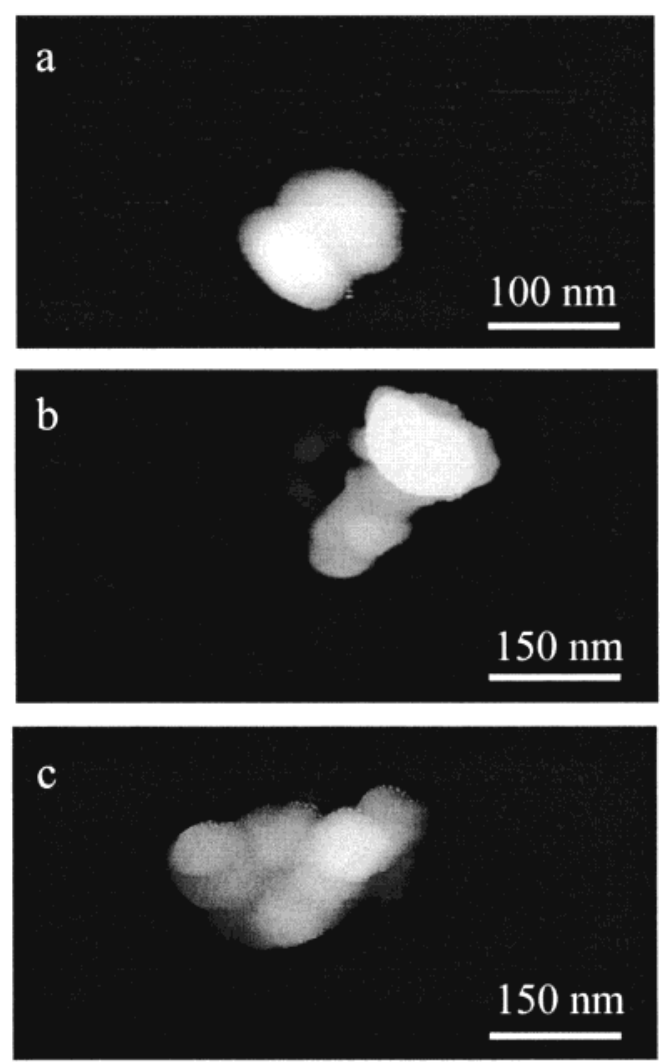

Figure 2. AFM images of 3 different SERS-active Ag aggregates prepared by incubating a $\mathrm{Ag}$ colloid with $10 \mathrm{mM} \mathrm{NaCl}$ and $2 \mathrm{nM} \mathrm{R6G}$. The heights of the aggregates ranged from $\sim 50$ to $\sim 200 \mathrm{~nm}$.

\section{Results}

Figure 1 shows a correlated single molecule R6G SERS spectrum, resonant Rayleigh scattering spectrum, and AFM image of an individual SERS-active $\mathrm{Ag}$ nanoparticle. The marked peaks in the SERS spectrum correspond to the Raman bands for R6G. Consistent with prior results, the resonant Rayleigh scattering spectrum shows some scattering intensity at $514.5 \mathrm{~nm}$, the laser excitation wavelength, and appears to have at least 2 resonances. The AFM image shows that this particular SERS-active particle is a tightly packed aggregate consisting of $\sim 12$ to 15 particles.

The large majority of $\mathrm{Ag}$ particles on the surface were isolated, single nanocrystals, yet all of SERS-active particles identified were compact clusters that consisted of a minimum of 2 individual Ag particles. Tapping mode AFM images of three additional SERS-active nanocrystals are shown in Figure 2. Each of these particles corresponds to a scattering spectrum with multiple peaks (not shown), verifying that the presence of multiple scattering resonances corresponds to aggregates. While the individual particles are $\sim 55 \mathrm{~nm}$ in diameter, the SERSactive aggregates range from $\sim 100$ to $\sim 250 \mathrm{~nm}$ in size and vary significantly in shape as well. These results are consistent with two recent reports. Xu et al. observed single molecule hemoglobin SERS only from dimers and trimers of colloidal Ag particles. ${ }^{14}$ Similarly, Moyer et al. observed SERS of single carbon domains from $75 \%$ of the Ag particles in their sample, all of which consisted of clusters of Ag crystallites. ${ }^{15}$

It is well-known that the addition of low concentrations of $\mathrm{Cl}^{-}$ions $(1-10 \mathrm{mM})$ to the $\mathrm{Ag}$ colloid yields significant increases in SERS signals. Since extinction spectra, TEM, and AFM measurements have shown that such low quantities of $\mathrm{Cl}^{-}$ions do not cause any significant aggregation of the sample, 


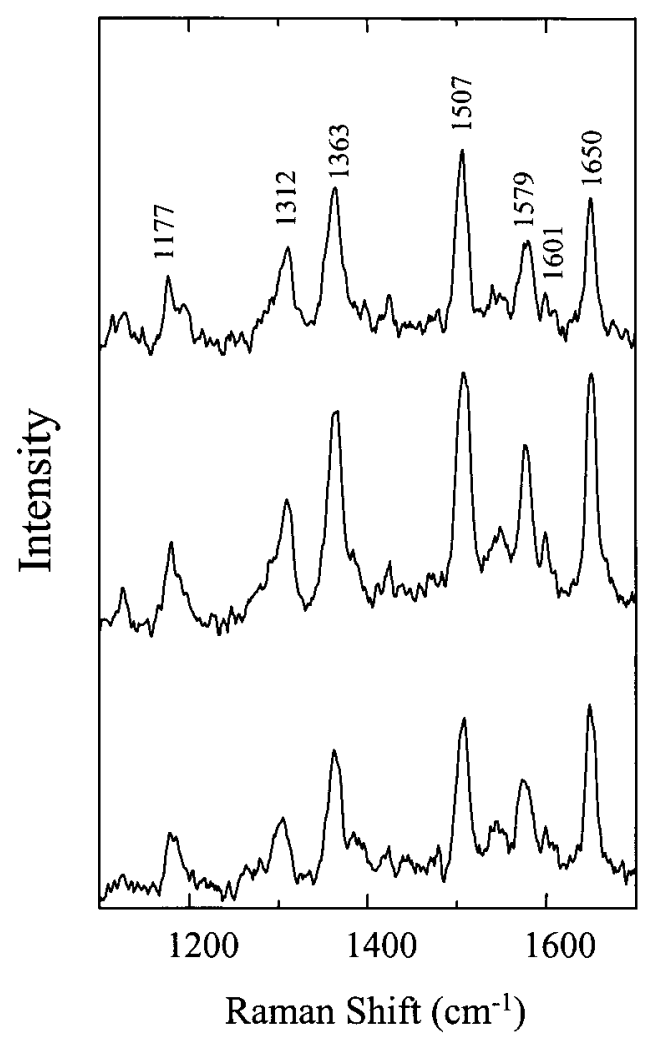

Figure 3. Three consecutive R6G SERS spectra obtained at $\sim 30$ $\mathrm{W} / \mathrm{cm}^{2}$ with $60 \mathrm{~s}$ integration time. The fwhm of the Raman lines ranges from $\sim 12$ to $15 \mathrm{~cm}^{-1}$. The magnitude of the change in relative intensities of the Raman bands is $\sim 20 \%$.

it has often been proposed that the anions serve as "activating agents". This activation may occur either by generating unique "SERS-active sites" on the nanocrystal or by stabilizing the chemisorbed molecule. ${ }^{5,16}$ To test the importance of a potential "anion effect", we screened for single molecule R6G SERS in a sample initially incubated with polylysine and ascorbic acid in place of $\mathrm{NaCl}$. Polylysine, in conjunction with ascorbic acid, is a well-known aggregating agent. ${ }^{17}$ For this sample, the same observations were noted as for $\mathrm{NaCl}$ : (1) only a small percentage of Ag particles exhibited SERS-activity, and (2) the scattering spectra of all such SERS-active particles were characterized by multiple resonances. This suggests that the addition of $\mathrm{NaCl}$ serves to induce aggregation in the colloid and provides further evidence that aggregates are indeed responsible for the enormous SERS enhancements.

These results strongly suggest that the special site for single molecule SERS, whose rarity leads to signal saturation at very low concentration in bulk colloids, lies at the junction of two Ag particles in a compact cluster. Classical electromagnetic calculations show that this junction, for Angstrom-scale separation of the two particles, experiences a significantly higher electromagnetic field and ballistic carrier surface flux than any other location on the surface of a single metal particle. We will return to this point in the theory section.

Each SERS-active aggregate gives a somewhat different Raman signature. As previously reported, some single molecule SERS signals blink or show intensity fluctuations on a second time scale under $\mathrm{cw}$ laser excitation, while others do not. ${ }^{9}, 11$ Additionally, the absolute intensity and relative ratio of the sharp R6G Raman lines to the underlying continuum vary from one aggregate to the next. Figure 3 shows three consecutive R6G SERS single molecule spectra taken with $60 \mathrm{~s}$ integration times for which the relative intensities of the Raman bands show

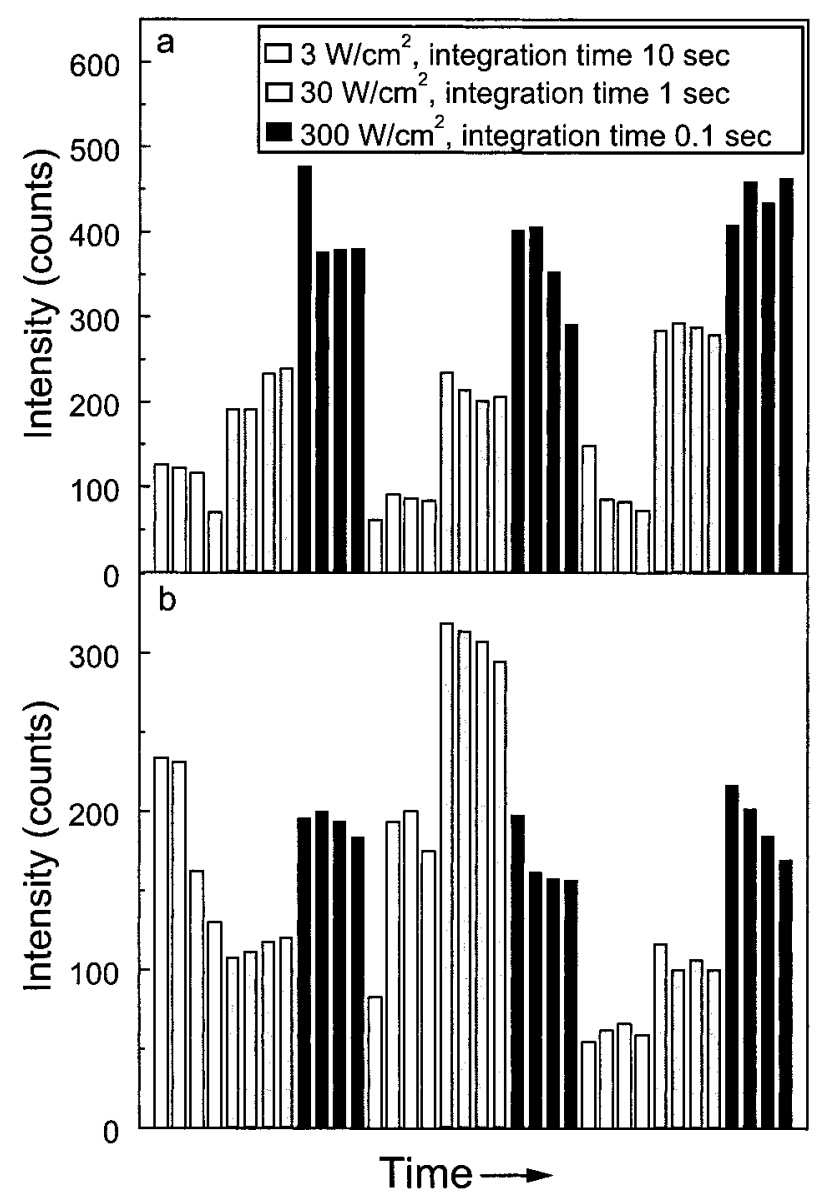

Figure 4. R6G SERS signal for two different Ag aggregates as a function of laser power under conditions of constant fluence. At each power, four consecutive signals were recorded to measure variations in the signal intensity as a function of time. For each particle, the power dependence was measured three consecutive times.

fluctuations of approximately $20 \%$. The spectral widths of these Raman lines range from $\sim 12$ to $15 \mathrm{~cm}^{-1}$. Spectra obtained with shorter integration times have shown significant frequency variations in the Raman signals as a function of time, therefore the fairly large width of these lines compared to classical Raman scattering may be partially accounted for by these spectral variations. ${ }^{9}$ Decreasing the integration time from 60 to $10 \mathrm{~s}$ in an attempt to eliminate the time-averaging of these spectral variations yields no noticeable change in the spectral width.

In addition to the varied behavior described above, the individual SERS-active aggregates also show different laser power dependencies. It is difficult to study the power dependence systematically in the presence of intensity fluctuations at constant laser power. Therefore, for a single SERS signal, we measure the integrated Stokes-shifted SERS intensity (including underlying continuum) for four consecutive periods at constant power, to obtain a measure of the signal reproducibility before changing laser power. Most remarkably, about $80 \%$ of the SERS-active aggregates showed greater scattering efficiency at higher powers, as shown in Figure 4. By increasing the laser power by a factor of 100 and decreasing the integration time by the same factor, we observed reproducible increases in SERS signals ranging from a factor of 4 to 6 . A small number of SERS signals exhibited a linear power dependence, and several showed decreases in efficiency with increasing power. Figure $4 \mathrm{~b}$ shows a SERS signal from a single particle where there is some fluctuation superimposed upon a varying power dependence over the course of the experiment. There is no 


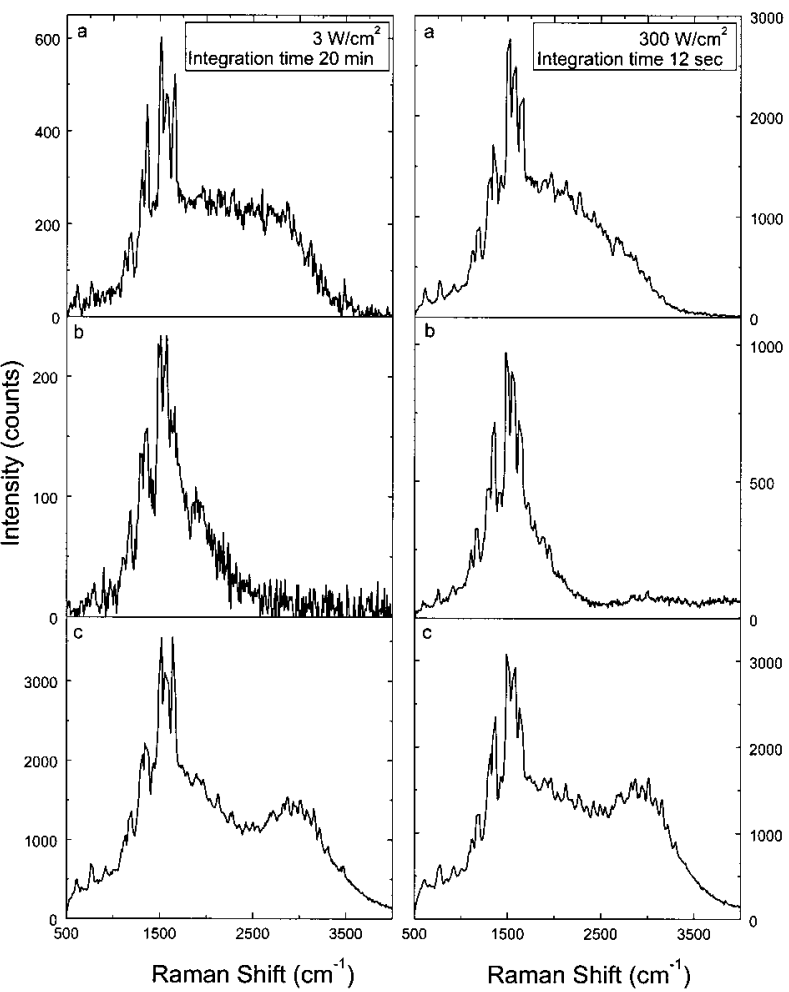

Figure 5. R6G SERS spectra at low power (left) and high power (right) for three SERS-active Ag aggregates.

apparent correlation between the laser intensity and the frequency of fluctuations. Despite this superlinearity, both the underlying continuum and the sharp Raman lines show a nearly identical power dependence, as shown in Figure 5 for three different SERS aggregates.

If the Raman species were a photoproduct, then one might expect superlinear behavior, but we see the same sharp Raman lines at low and high power. We also measured the $514.5 \mathrm{~nm}$ Rayleigh scattering for the same time periods and laser powers for particles throughout the entire sample. Within a reproducibility of about 20\%, the Rayleigh scattering is linear, as expected. Thus the vibronic coupling between the resonant optical state and the molecular vibration is stronger at higher optical fields. To our knowledge, this type of superlinearity has never been seen in a Raman experiment.

We explored the SERS excitation spectrum by comparing SERS activity with 514.5 and $632.8 \mathrm{~nm}$ laser excitation. As previously stated, at nanomolar concentrations of R6G, approximately 1 out of every $200 \mathrm{Ag}$ particles yields single molecule SERS, when the sample is incubated with $10 \mathrm{mM}$ $\mathrm{NaCl}$ and excited at $514.5 \mathrm{~nm}$. In one sample, using $514.5 \mathrm{~nm}$ excitation, 32 such SERS-active particles were identified, all of whose spectra consisted of sharp R6G Raman lines superimposed upon a broad continuum. All strong SERS signals contain both continuum and sharp Raman lines; occasionally signal at $10 \times$ weaker is observed consisting only of continuum. When the excitation wavelength was changed to $632.8 \mathrm{~nm}, 10$ of these particles showed significant Stokes shifted intensity. However, 9 of these 10 particles showed only continuum emission without any evidence of R6G Raman lines under 632.8 $\mathrm{nm}$ exctitation, while the remaining one particle showed both R6G Raman and continuum emission. With $632.8 \mathrm{~nm}$ excitation, no new particles exhibited SERS activity that were not part of the $514.5 \mathrm{~nm}$ active group. When the laser wavelength was returned to $514.5 \mathrm{~nm}$, the original SERS activity remained in these 32 particles.
This result indicates that a Ag aggregate that yields both Raman and Stokes-shifted continuum emission under $514.5 \mathrm{~nm}$ excitation has a $1 / 3$ probability of emitting a broad continuum alone (no Raman lines) when excited at $632.8 \mathrm{~nm}$. The excitation spectrum of the underlying SERS continuum therefore appears to differ, in this limited sample, from that of the R6G Raman scattering, by extending further red in wavelength. The continuum excitation spectra may, in fact, more closely follow the resonant Rayleigh scattering spectrum. While Rayleigh scattering spectra were not acquired in this experiment, many of the 32 SERS-active particles likely had strong resonances at $632.8 \mathrm{~nm}$ based upon results of our prior studies, which showed that the scattering spectra of SERS-active particles often extend over the entire visible spectrum. Since excitation at $632.8 \mathrm{~nm}$ lies below the absorption band of aqueous R6G (not chemisorbed) which peaks at $530 \mathrm{~nm}$, but still lies well within the plasmon resonance of many of the $\mathrm{Ag}$ particles present in the sample, this result suggests that HOMO-LUMO resonance of the molecule plays a crucial role in yielding sharp R6G Raman lines. This observation is also consistent with colloidal SERS study by Hildebrandt and Stockburger, which reported the excitation spectrum of the giant SERS signal to follow the absorption spectrum of R6G. ${ }^{5}$ Yet, while the Raman and underlying continuum emission appear to originate from two different spectroscopic processes, both show the same superlinear dependence and are clearly linked. If the signal blinks, both the continuum and Raman lines blink on and off together.

\section{Raman Scattering Theory}

Large Ag particles more than $30 \mathrm{~nm}$ diameter have huge electronic transition dipoles with femtosecond radiative widths and negligible vibronic coupling to low frequency Ag normal modes. Such particles show intense Rayleigh scattering without any Stokes shifted emission. A very simple theoretical approach to SERS is to assume that chemisorption vibronically couples the R6G vibrations to the plasmon transition dipole, without specifying the microscopic nature of this coupling. This can be done by treating the $\mathrm{Ag} /$ molecule system in the framework of conventional molecular electronic resonance Raman theory, which is well developed for biological chromophores of high oscillator strength. ${ }^{18,19}$ When the laser wavelength is resonant with an electronic absorption band, the intensities of the Raman lines are determined primarily by the properties of the excited electronic state. In particular, the coupling of the vibrational modes of a molecule to its electronic state can be described as a function of a dimensionless displacement, $\Delta$, between the ground and excited electronic potential energy surfaces along a single normal coordinate. For resonant scattering, the total cross section for a Raman transition from initial state $|i\rangle$ to final state $|\mathrm{f}\rangle$, in the Born-Oppenheimer and Condon approximations, can be written as ${ }^{18}$

$$
\sigma_{\mathrm{i} \rightarrow \mathrm{f}}\left(E_{\mathrm{L}}\right)=\frac{8 \pi}{9 \eta^{2} c^{4}}\left(E_{\mathrm{S}}\right)^{3} E_{\mathrm{L}}\left|\alpha_{\mathrm{i} \rightarrow \mathrm{f}}\right|^{2}
$$

where

$$
\alpha_{\mathrm{i} \rightarrow \mathrm{f}}=M^{2} \sum_{\mathrm{v}} \frac{\langle\mathrm{f} \mid \mathrm{v}\rangle\langle\mathrm{v} \mid \mathrm{i}\rangle}{\epsilon_{\mathrm{v}}-\epsilon_{\mathrm{i}}+\mathrm{E}_{0}-\mathrm{E}_{\mathrm{L}}-\mathrm{i} \Gamma}
$$

The cross section $\sigma$ is in units of area, $E_{\mathrm{L}}$ and $E_{\mathrm{S}}$ are the energies of the incident and scattered photons, and $\alpha_{\text {if }}$ is the resonance Raman polarizability transition matrix element between states $|i\rangle$ and $|f\rangle$ as given by the Kramers-Heisenberg-Dirac dispersion 
formula. In eq 2, $M$ is the electronic transition dipole moment; $|\mathrm{v}\rangle$ is a vibrational level of the excited electronic state; $\epsilon_{\mathrm{v}}$ and $\epsilon_{\mathrm{i}}$ are the energies of the states $|\mathrm{v}\rangle$ and $|\mathrm{i}\rangle$, respectively; $E_{0}$ is the energy difference between the lowest vibrational levels of the ground and excited electronic states; and $\Gamma$ is the homogeneous line width of the electronic transition. The terms $\langle\mathrm{f} \mid \mathrm{v}\rangle$ and $\langle v \mid i\rangle$ are the appropriate Franck-Condon overlap integrals. Assuming that both the excited and ground state potential energy surfaces are harmonic and differ only in their equilibrium position, $\Delta$, the overlap integrals for Rayleigh, fundamental Raman, and overtone scattering, respectively, are given by ${ }^{20}$

$$
\begin{gathered}
\langle 0 \mid \mathrm{v}\rangle\langle\mathrm{v} \mid 0\rangle=\frac{\Delta^{2 \mathrm{v}}}{2^{\mathrm{v}} \mathrm{v} !} \exp \left(-\Delta^{2} / 2\right) \\
\langle 0 \mid \mathrm{v}\rangle\langle\mathrm{v} \mid 1\rangle=\langle 0 \mid \mathrm{v}\rangle\langle\mathrm{v} \mid 0\rangle\left(\Delta-\frac{2 \mathrm{v}}{\Delta}\right) / \sqrt{2} \\
\langle 0 \mid \mathrm{v}\rangle\langle\mathrm{v} \mid 2\rangle=\langle 0 \mid \mathrm{v}\rangle\langle\mathrm{v} \mid 0\rangle\left(\Delta^{2}-4 \mathrm{v}+4 \mathrm{v}(\mathrm{v}-1) / \Delta^{2}\right) / 2 \sqrt{2}
\end{gathered}
$$

We consider a spherical particle with a diameter of $100 \mathrm{~nm}$ and which is characterized by the optical dielectric constants of bulk Ag. Mie theory calculations show that the scattering resonance peaks at $397 \mathrm{~nm}$ with a fwhm of $\sim 90 \mathrm{~nm} .{ }^{21}$ As the scattering cross section is far larger than the absorption cross section, the large spectral width of the resonance corresponds directly to the femtosecond radiative decay rate $\Gamma$. Relating the peak intensity and spectral width of the resonance to the transition dipole shows that for a particle that is $100 \mathrm{~nm}$ in diameter, $M$ approaches a remarkable $21000 \mathrm{D}$.

For the $1575 \mathrm{~cm}^{-1} \mathrm{C}-\mathrm{C}$ aromatic stretch for R6G, the experimentally measured Rayleigh scattering and SERS cross sections agree with the values calculated for $\Delta=0.012$ and the values of $E_{0}$ and $\Gamma$ calculated from Mie theory as described above. At an excitation wavelength of $514.5 \mathrm{~nm}$, the model predicts a fundamental Raman scattering cross section of 1.5 $\times 10^{-15} \mathrm{~cm}^{2}$ and a Rayleigh scattering cross section of $\sim 2 \times$ $10^{-10} \mathrm{~cm}^{2}$. Assuming that the sharp $1575 \mathrm{~cm}^{-1}$ band contributes approximately $5 \%$ of the total measured SERS cross section of $2 \times 10^{-14} \mathrm{~cm}^{2}$, the measured SERS cross section for this band is $1 \times 10^{-15} \mathrm{~cm}^{2}$. The measured Rayleigh scattering cross section, which is approximately 6000 times larger than the total SERS cross section, is $\sim 1 \times 10^{-10} \mathrm{~cm}^{2}$. While there have been no reports to date of overtones observed in single molecule SERS spectra, ensemble studies have measured that the signals from fundamental Raman scattering of R6G SERS are approximately 10 times stronger than the overtone signals. ${ }^{5}$ The resonance Raman model predicts that the ratio of fundamental Raman scattering to overtone scattering for this system should approach 6 orders of magnitude and is consistent with the fact that overtones have not been observed at the single molecule level.

The excited state of the particle is very weakly coupled to the molecule, yet the Raman cross section is so large because the Ag transition dipole is huge. The Raman scattering is similar to resonance Raman scattering from a dissipative continuum in a molecular system, because the excited state dephases so quickly. Despite this insight, the model is too simple because the Raman excitation spectrum (eq 1) is predicted to be the same as the resonant Rayleigh scattering spectrum for the Ag particle. Our limited data examining the nature of the excitation profiles of the particles indicate the true situation is more complex.

The plasmon excited state is a correlated, many-electron excitation, which Kawabata and Kubo originally described as

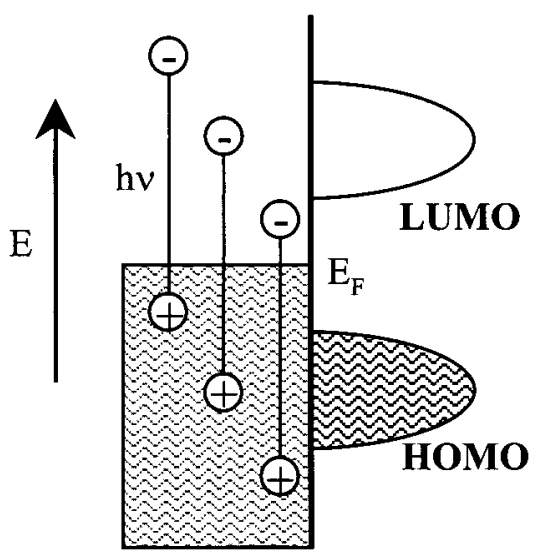

Figure 6. Schematic of relative energies of excited electron-hole pairs generated via excitation of the surface plasmon in the metal with respect to the HOMO and LUMO of the chemisorbed molecule.

a coherent sum of all possible electron-hole excitations, for which the energy separation of each pair corresponds to the laser frequency. ${ }^{22}$ The molecular Raman model described above essentially treats vibrational coupling to all components of this wave function equivalently. If some components couple more strongly to the molecule than others, then the resonant state is different than the zero order plasmon state and a more complex model is required. In Otto's and Persson's models, individual ballistic electrons or holes generated via excitation of the surface plasmon couple to the chemisorbed molecule LUMO or HOMO as shown in Figure 6. In this description, the relative energy of the HOMO and LUMO with respect to the Ag Fermi level is critical. If the LUMO is too high in energy with respect to the Fermi level, or the HOMO similarly lies too low in energy, neither the excited electrons or holes in the metal can efficiently couple to the molecule. The interaction between the metal and molecule will be maximized when the HOMO-LUMO separation is resonant with the excitation wavelength and symmetric with respect to the Ag Fermi level.

This coupling between the metal electrons and chemisorbed molecules clearly occurs. In Ag particles, the dephasing rate of the surface plasmon increases in the presence of chemisorbed molecules which have low-lying LUMO levels that can interact with ballistic electrons in the metal. ${ }^{23}$ The faster decay rate occurs due to scattering of metal electrons off the chemisorbed molecules via transient negative ion formation. ${ }^{24}$ Similarly, DC resistivity measurements have shown that only those molecules that yield a change in resistivity upon adsorption to the metal contribute to the SERS signal. In other words, only those molecules with low-lying LUMOs that can interact with the metallic electrons yield an observable SERS signals. ${ }^{1}$ This type of resonance may be the origin of reported single molecule SERS in the near-IR.

A second interaction mechanism between the metal and molecule involves exchange mixing of Ag neutral electronhole pairs with the HOMO-LUMO neutral excited state of the chemisorbed molecule. This mechanism would yield a neutral excited R6G type resonant state, rather than a charged resonant molecular state. As the photon energy becomes resonant with the HOMO-LUMO gap, mixing should increase. If all pairs equally mix with the neutral excited molecular state at a given photon energy, independent of the position of the Fermi level, then this idea is mathematically equivalent to $\Delta$ being wavelength dependent. A wavelength-dependent $\Delta$ is not a useful physical concept, yet such behavior seems to appear in the single molecule SERS experiment, as the SERS excitation spectrum is similar to the aqueous R6G absorption spectrum. Possible 
further evidence for this mechanism is the observation that the R6G vibrations observed in single molecule SERS are nearly identical to the modes observed in R6G resonance Raman scattering. 5

\section{Nanocrystal Junctions as the Site for Single Molecule SERS}

Why is the junction between two large Ag nanocrystals a site for single molecule SERS? As two large Ag particles approach each other, their transition dipoles, composed of oscillating, ballistic carriers in each particle, couple. Classical electromagnetic theory predicts that the enhanced fields around each particle coherently interfere. As the distance between the nanocrystals decreases, the coupled plasmon resonance shifts to the red, the enhanced electromagnetic field increases in the junction between the particles, and destructive interference of the fields occurs at other points in space. For a gap of $1 \mathrm{~nm}$ between two spheres that are $90 \mathrm{~nm}$ in diameter, the SERS field enhancement factor, $\left(\mathbf{E} / \mathbf{E}_{\text {laser }}\right),{ }^{4}$ is $\sim 10^{10}$ in a volume of space that could contain only a few molecules. ${ }^{14}$ At $30 \mathrm{~W} / \mathrm{cm}^{2}$, the field intensity in the gap would be $3 \times 10^{6} \mathrm{~W} / \mathrm{cm}^{2}$. The junction can therefore function as an electromagnetic "hot spot" analogous to those predicted to exist in large fractal aggregates of Ag particles. ${ }^{25,26}$

This example shows that resonant Rayleigh scattering spectra are limited in their use as a probe of the electromagnetic field enhancement around an individual particle. The Rayleigh scattering intensity measures the square of transition dipole of the particle, the same quantity which creates the enhanced local field. As the dipole increases, the local field increases as well. While the dipole is a measure of the total integrated field energy density around the particle, it provides no information about the spatial distribution of the local field. However, when the Rayleigh spectrum contains multiple resonances of similar intensities, the particle must be an aggregate, and thus likely has regions of high field intensities at the junctions.

Gauss' Law describes a direct link between the enhanced field and the local flux of ballistic electron-hole pairs incident upon a molecule chemisorbed at the junction when the laser polarization is along the dimer axis. Since the dimensions of the junction are small $(d \ll \lambda)$, electric fields and charges can be considered in the electrostatic limit. The discontinuity in enhanced field $\mathbf{E}$ along the dimer axis is $\sigma / \epsilon_{0}$, where $\sigma$ is the incident ballistic charge density during one-half of an optical cycle. Garcia-Vidal and Pendry have presented an analogous effect for the junction between two touching Ag cylinders. ${ }^{27}$ As the particles approach each other, $E$, the flux of carriers, and equivalently, the concentration of coherent pairs at the junction, all increase. The capacitive coupling of one Ag particle to the other refocuses the coherent current density of ballistic carriers onto the junction surface: these carriers create the field in the junction.

As two $55 \mathrm{~nm} \mathrm{Ag}$ particles approach each other, there is a large van der Waals attraction. One could imagine that untethered particles would approach until the surface chemisorbed layers on both particles merge. It is possible that a chemisorbed R6G molecule in the junction may electrically contact both Ag particles. We might conjecture that this system operates as a molecular tunnel junction, where hot electrons tunnel between the two metal particles across a vibrating molecular bridge. This type of system strikingly resembles two different experiments that have been reported in the recent literature. In STM vibrational spectroscopy, a metal tip is brought within several angstroms of a molecule chemisorbed to a metal surface. ${ }^{28}$ An increase in conductance is measured when the energy of the injected electrons reaches the energy of a normal mode of the molecule due to the onset of a previously forbidden inelastic tunneling channel. Similarly, DC conductance studies of molecular wires are examinations of electron transport across metal electrode - molecule - metal electrode junctions. ${ }^{29}$

The laser intensity dependence of the vibronic coupling of the molecule to the resonant state is curious. Aqueous Rhodamine 6G molecules have been observed to move toward the laser focus due to the gradient force..$^{30}$ In our experiment at $30 \mathrm{~W} / \mathrm{cm}^{2}$, the enhanced electric field, and thus the biasing potential at the particle junction is at the same order of magnitude as reported. ${ }^{30}$ Yet, at the particle junction, the electric field, illustrated by touching cylinder model, ${ }^{27}$ falls off much faster than in the case of a focused laser beam. Thus, the gradient force at particle junctions is at least 100 times larger than as in the case of ref 30. As the laser intensity increases, the molecule will experience a greater force pulling it farther into the junction. Also, electron exchange may increase as the molecule is pushed into the metal surface. At these high intensities, it is also possible that an optical Stark effect shifts the energy of the molecular states.

Each SERS-active R6G/Ag particle complex shows unique kinetic and spectroscopic behavior that must depend on the specific characteristics of the active site in the junction. The Raman signal and background continuum, while intimately linked, apparently have different excitation spectra. We suggest that the Raman process results from scattering off a resonant state made by mixing of $\mathrm{Ag}$ neutral ballistic pairs and the R6G neutral excited state as discussed above. We might conjecture that the background continuum results from plasmon dephasing via transient R6G ion formation. As discussed in our previous paper, ${ }^{11}$ a transient charge just above the metal surface in large Ag particles is known to excite broad metal luminescence.

Acknowledgment. We thank J. Trautman, T. D. Harris, J. Macklin, Al.A. Efros, J. Tully, and T. Heinz for informative discussions. We also thank the W. M. Keck Foundation for a start-up grant at Columbia, and Bell Laboratories for apparatus donation. This research was supported under DOE Grant DEFG02-98ER14861. Materials research facilities at Columbia University are supported under NSF MRSEC Grant DMR-9809687.

\section{References and Notes}

(1) Otto, A.; Mrozek, I.; Grabhorn, H.; Akemann, W. J. Phys.: Condens. Mater. 1992, 4, 1143.

(2) Moskovits, M. Rev. Mod. Phys. 1986, 57, 783.

(3) Schatz, G. Acc. Chem. Res. 1984, 17, 370.

(4) Campion, A. Chem. Soc. Rev. 1998, 4, 241

(5) Hildebrandt, P.; Stockburger, M. J. Phys. Chem. 1984, 88, 5935.

(6) Kneipp, K.; Wang, Y.; Kneipp, H.; Itzkan, I.; Dasari, R. R.; Feld, M. S. Phys. Rev. Lett. 1996, 76, 2444.

(7) Kneipp, K.; Wang, Y.; Kneipp, H.; Perelman, L. T.; Itzkan, I.; Dasari, R. R.; Feld, M. S. Phys. Rev. Lett. 1997, 78, 1667.

(8) Kneipp, K.; Kneipp, H.; Kartha, B.; Manoharan, R.; Deinum, G.; Itzkan, I.; Dasari, R. R.; Feld, M. S. Phys. Rev. E 1998, 57, R6281.

(9) Nie, S.; Emory, S. Science 1997, 275, 1102.

(10) Pettinger, B.; Krischer, K.; Ertl, G. Chem. Phys. Lett. 1988, 151, 151.

(11) Michaels, A. M.; Nirmal, M.; Brus, L. E. J. Am. Chem. Soc. 1999, 121,9932 .

(12) Persson, B. N. J. Chem. Phys. Lett. 1981, 82, 561.

(13) Otto, A. Surface-enhanced Raman Scattering: "Classical" and "Chemical" Origins. In Light Scattering in Solids IV; Cardona, M., Gundtherodt, G., Eds.; Springer-Verlag: Berlin, 1984; p 289.

(14) Xu, H.; Bjerneld, E. J.; Kall, M.; Borjesseon, L. Phys. Rev. Lett. 1999, 83,4357

(15) Moyer, P. J.; Schmidt, J.; Eng, L. M.; Meixner, A. J. J. Am. Chem Soc. 2000, 122, 5409 .

(16) Schneider, S.; Grau, H.; Halbig, P.; Freunscht, P.; Nickel, U. J. Raman Spectrosc. 1995, 27, 57. 
(17) Munro, C. H.; Smith, W. E.; Armstrong, D. R.; White, P. C. J. Phys. Chem. 1995, 99, 879.

(18) Myers, A.; Mathies, R. Resonance Raman Intensities: A Probe of Excited-State Structure and Dynamics. In Biological Applications of Raman Spectroscopy; Spiro, T. G., Ed.; Wiley \& Sons: New York, 1987; Vol. 2; p 1.

(19) Myers, A. B. Excited Electronic State Properties From GroundState Resonance Raman Intensities. In Laser Techniques in Chemistry; Myers, A. B., Rizzo, T. R., Eds.; John Wiley \& Sons: New York, 1995; Vol. 23; p 325

(20) Chinsky, L.; Laigle, A.; Peticolas, W. L.; Turpin, P.-Y. J. Chem. Phys. 1982, 76, 1.

(21) Wickramasinghe, N. C. Light Scattering by Spherical Particles; Wiley: New York, 1973; p 25.
(22) Kawabata, A.; Kubo, R. J. Phys. Soc. Jpn. 1966, 21, 1765.

(23) Kreibig, U.; Gartz, M.; Hilger, A. Ber. Bunsenges. Phys. Chem. 1997, 101, 1593.

(24) Persson, B. N. J. Surf. Sci. 1993, 281, 153.

(25) Markel, V. A.; Shalaev, V. M.; Zhang, P.; Huynh, W.; Tay, L.; Haslett, T. L.; Moskovits, M. Phys. Rev. B 1999, 59, 10903.

(26) Bozhelvolnyi, S. I.; Markel, V. A.; Coello, V.; Kim, W.; Shalaev, V. M. Phys. Rev. B 1998, 58, 11441.

(27) Garcia-Vidal, F. J.; Pendry, J. B. Phys. Rev. Lett. 1996, 77, 1163

(28) Stipe, B. C.; Rezaei, M. A.; Ho, W. Science 1998, 280, 1732.

(29) Yaliraki, S. N.; Kemp, M.; Ratner, M. A. J. Am. Chem. Soc. 1999, $121,3248$.

(30) Osborne, M. A.; Balasubramanian, S.; Furey, W. S.; Klenerman, D. J. Phys. Chem. B 1998, 102, 3160. 